\title{
THE EFFECT OF OSSEODENSIFICATION TECHNIQUE ON IMPLANT STABILITY (CLINICAL TRIAL)
}

\author{
Ahmed M. Ibrahim ${ }^{*}{ }_{B D S}$, Sherif S. Ayad ${ }^{2}$ Ph.D., Adham ElAshwah' Ph.D.
}

\section{ABSTRACT}

INTRODUCTION: Dental implants primary or initial stability originates from the engagement with the cortical bone mechanically, whereas the secondary stability signifies the stability biologically by means of osseointegration through the bone formation and remodeling from the cancellous bone. Osseodensification is an innovative biomechanical technique to prepare the site. It uses Densifying Burs to produce low plastic deformation owing to its non-removal densifying method of site preparation, which preserves the bone enhancing the host site.

OBJECTIVES: To evaluate the osseodensification (OD) technique used in implant site preparation by using the newly designed burs (Densah burs), and its effect on enhancing implant primary and secondary stability.

MATERIALS AND METHODS: Twenty dental implants were placed in ten patients; each patient received one implant using the new Osseodensification drilling technique and one implant using the conventional drilling technique in the posterior maxillary ridge. Osstell was used in measuring resonance frequency (ISQ-scale) which is used to determine the amount of implant stability.

RESULTS: The results showed a significant improvement in both primary and secondary stability using densah burs.

Conclusion: Densah burs produce better bone quality around the implant than conventional drills, and therefore, there is an improvement in the primary and secondary stability.

KEY WORDS: Osseodensification, Densah, Dental Implants, Implant Stability, in vitro.

RUNNING TITLE: The effect of osseodensification technique on implant stability

1 BDS, Faculty of Dentistry, Alexandria University

2 Professor of Oral and Maxillofacial Surgery, Faculty of Dentistry Alexandria University

3 Lecturer of Oral and Maxillofacial Surgery, Faculty of Dentistry, Alexandria University

* Corresponding Author:

E-mail: a.medhat.1991@gmail.com

\section{INTRODUCTION}

Dental implants primary or initial stability originates from the engagement with the cortical bone mechanically, whereas the secondary stability signifies the stability biologically by means of osseointegration through the bone formation and remodeling from the cancellous bone.(1)

The primary stability is considered the substantial factor for secondary stability. Primary stability depends on the surgical technique (surgeon factor), bone quality \& quantity (patient factor), and the implant design and characteristics (implant factor). On the other hand, secondary stability depends on primary stability, bone modeling and remodeling, and implant surface treatment.(1)

Bone density of poor quality is quite common in the human maxilla, particularly in old patients requiring a fixed implantsupported treatment. In D3 or D4 bone type, it is hard to complete a good implant primary stability because of the poor bone value percentage (\%BV) around the titanium implant surface.(2) Consequently, it is difficult to achieve a high implant primary stability. (3)

Several clinical researches recommend placing implants immediately after tooth extraction which may offer some advantages, as reducing bone resorption, sustaining alveolar crest width and height, lessening surgical procedures and treatment time and, lastly, good esthetic outcomes, as the implant can be placed along with the natural tooth angulation and aligned with the adjacent teeth. This procedure has been demonstrated to have a high clinical success rate after more than one year. Immediate implant placement after tooth extraction is frequently accompanied by a remaining gap between the coronal part of the implants and residual bone walls. (4)

If the primary stability of the implant is insufficient, the early implant failure rate could escalate beyond critical levels. Immediate loading procedures are also not encouraged in the event of low primary implant stability or poor bone quality. Besides, extended healing time is required in these situations, with a few disadvantages for the patient. (5)

Numerous surgical procedures have been suggested to avoid or diminish bone loss during implant placement in low-density bone and to improve implant's primary stability and bone quality. Few researchers advised diminishing the osteotomy site in comparison to the implant diameter of approximately $10 \%$ to decrease bone cutting and improve implant's primary stability. An osteotomy site diminished more than $10 \%$ did not provide mechanical benefit. However, this expedient allows to increase implant primary stability. (6-9)

Standard drills used in implantology are made to create room by excavating bone for implant placement. They excavate bone 
efficiently however they normally do not produce a precise circumferential osteotomy. (10)

Osseodensification is an innovative biomechanical technique to prepare the site. It uses Densifying Burs to produce low plastic deformation owing to its non-removal method of site preparation, which preserves the bone enhancing the host site. The densifying bur rotates at $800-1500$ RPM in the counterclockwise non-cutting direction (Densifying mode). They can also rotate clockwise (Cutting Mode) to cleanly cut bone if required. This dual use capability permits clinical versatility. (10) Osseodensification does not excavate bone, opposing traditional bone drilling techniques. It rather preserves the bulk of the bone. Consequently bone tissue is compacted in an externally expanding direction to form the osteotomy. (10)

Osseodensification technique requires adequate irrigation to evade heat generation. Consequently, in guided surgeries, it is vital to choose a guided system that utilizes an open guide to avoid hindering irrigation flow. The pilot drill (simple twist drill) is advanced to the prearranged depth in the right direction in the traditional way. The rest of the procedure is to some extent reliant on the site. There are tapered and parallel sided drills for use with tapered implants for use with parallel implants. The full kit of 13 Densah drills (Versah) can be used with most implant systems.(11)

The rationale behind this process is the densification of the bone that will be in immediate contact to the implant results in higher degrees of primary stability due to physical interlocking between the bone and the device, faster new bone growth formation due to osteoblasts nucleating on instrumented bone that is in proximity with the implant.(12)

The Densah Burs can also rotate in a cutting clockwise motion (Cutting Mode) to efficiently remove bone if required. This dual ability permits clinical resourcefulness. it may permit the implant surgeon to autograft the maxillary sinus and proficiently expands any ridge in either jaw with improved implant stability. (13)

There are drilling procedures for each implant type and bone density. The decision to either cut or densify is reliant on bone density. Cutting is attained by running the drills in a clockwise motion at 800 to 1500 rpm.(11)

The osseodensification procedure can be used any place the bone is adequately plastic to be densified. This procedure can work particularly well underneath the maxillary sinus, where it is made possible to raise the sinus floor without violating the sinus and increase vertical height.(11)

The current study aims to evaluate the effect of osseodensification on implant primary and secondary stability.

\section{MATERIALS AND METHODS}

\section{Study design}

The study was a randomized controlled clinical trial performed on 10 patients requiring two dental implants in the posterior maxillary ridge. Both genders were selected of age range from 20 to 50 years, from those seeking dental implants and attending the outpatient clinic of the Oral and Maxillofacial Surgery Department, Faculty of dentistry, Alexandria University.
The study was performed after gaining the approval of the Research Ethics Committee, Faculty of Dentistry, Alexandria University. Biosafety principles were adapted during the implant placement where patients went under medical investigations before surgery.

Informed consent was taken from all patients after explaining all the procedures to the patient including all benefits and side effects simply and easily. Also, the patients had the right to withdrawal at any time.

Materials used

The materials that was used in this study are:

- Implant system (Dentium Co Ltd: \#214, 105, Gwanggyo-ro, Yeoungtong-gu, Suwon-si, Gyeonggi-do, Korea Tel: +82-31888-5431 www.dentium.com): two-piece implant (10 mm in length and $4 \mathrm{~mm}$ in diameter) with conical hex implant/abutment connection, round tapered design and implant healing abutments.

- Osseodensification densah burs (Versah, LLC: 2500 West Argyle Street, Suite 300 Jackson, Michigan 49202 Tel: 844711-5585 www.versah.com).

- Osstell ISQ-mentor device and Smart pegs (Osstell AB, Stampgatan 14, 41101 Göteborg, Sweden phone: Tel: +46 31 3408250 www.osstell.com)

\section{Methods}

The sample size was estimated by sample site calculation done in Medical Research Institute, Alexandria University.

In the same patient, split mouth technique was used. One implant site was prepared using the osseodensification burs technique (test side) and the other was prepared using the conventional burs technique according to the manufacturer guidance (control side). Implant stability quotients (ISQ) was measured immediately after implant placement and 4 months post placement.

Inclusion criteria

1. Adequate oral hygiene.

2. Patients between 20 and 50 years old.

3. Patients with at least two missing teeth in the posterior maxillary ridge

4. Proper interocclusal space in posterior maxillary region

5. Sufficient regenerated gingiva

Exclusion criteria

1. Heavy Smokers more than 10 cigarettes per day.

2. Patients with medically compromised diseases affecting placement of implant.

3. Current chemotherapy or radiotherapy.

4. Alcohol or drug abuse.

5. Presence of uncontrolled periodontal diseases.

6. Pregnant and lactating women.

7. Patients on Oral contraceptive pills

8. Patients suffering from osteoporosis

9. Sites with acute infection.

10. Sites requiring bone grafting.

11. Parafunctional habits 
Pre-Surgical Assessment

A- History of the patient

- Personal history: data was collected and recorded in full details including name, age, and occupation, address and telephone number.

- Past medical history.

- Past dental history: including the cause of extraction.

B- Clinical examination

It was performed both extra orally and intraorally. (14)

C- Radiographic examination

A routine panoramic x-ray was done followed by cone beam computed tomography (CBCT) to evaluate bone quality, width and height at the site of operation. (15)

Surgical phase and primary stability measurement:

- The oral cavity was prepared using $0.12 \%$ chlorohexidine mouth rinse for 30 seconds.

- Local anesthesia 2\% lidocaine (1:100000 epinephrine) (Amoun Pharmaceutical - Egypt) was administered at the site of operation.

- Horizontal mid-Crestal incision with full-thickness flap reflection to expose the surgical site using a sharp periosteal elevator. (16)

- Each patient received two implants in the maxilla, one on each side.

- One implant site was prepared using conventional drills according to the manufacturer guidance under profuse saline irrigation (control side). The other implant site was placed using the osseodensification Densah burs, under profuse saline irrigation with the following protocol (test side).

o First drill (pilot drill) to the full length, drill will rotate in clockwise rotation at rpm 800-1200, sequential using of the next drills rpm $800 \quad 1500$ anti-clockwise (non-cutting densifying mode) in a bouncing motion up and down to full depth till adequate diameter is reached. (figure 1)

o After reaching the required diameter, (figure 2) implant was carried out using hand driver and placed in the osteotomy socket then torque wrench was used on top of the hand driver to place the implant to the full depth inside the socket (figure 3)

- After implant placement, the smart peg was connected to the implant, by using osstell mentor Buccal-lingual ISQ readings was obtained.

- The flap was repositioned and sutured by 3/0 silk suture material (Johnson \& Johnson, New Jersey, USA)

Post-Surgical Phase

A- Post-operative care:

- All patients were instructed to apply cold fomentation starting immediately and for the following $24 \mathrm{hrs}$.

- Oral hygiene recommendation.

Antibiotics (Augmentin ${ }^{\circledR}$ - GlaxoSmithKline - Australia)

(Amoxicillin + clavulanic acid 1 gm.- Sigma Pharmaceutical

Industries - Mounfia - Egypt) every 12 hours for five days. (17)

- Non-steroidal anti-inflammatory drugs (Cataflam (Diclofenac potassium $50 \mathrm{mg}$ ) Novartis pharma, AG, Basel, Switzerland) every eight hours for five days.

- $0.12 \%$ chlorohexidine mouth rinse for seven days.
B- Wound healing

The sutured wound was examined for signs and symptoms of infection including swelling, redness, hotness, pus discharge, and pain in addition to observation for any manifestations of wound healing disturbance, as wound dehiscence. Sutures were removed one week after surgery.(18)

C- Stability of implants

A healing abutment was removed, and the smart peg was connected to the implant, by using osstell mentor Buccallingual ISQ readings was obtained again after implant placement 4-month post placement. Each smart peg was removed from its correspondent implant, chemically disinfected, and stored in sterilization pouch with all patient's data on it. $(19,20)$

D- Final prosthesis

Final prosthesis was constructed and delivered to the patient after 6 months.

Data Management \& Statistical Analysis

The data were processed and analyzed using the Statistical Package for Social Sciences program SPSS (15.0) software (SPSS Inc., Chicago IL, USA). The study included descriptive and analytical data. A P-value of less than 0.05 was considered statistically significant.

\section{RESULTS}

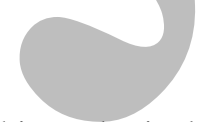

The demographical data of this study; including the gender and age of the participants were as follows:

- 5 males and 5 female patients participated in this study

- The ages of the participants ranged from 31.0 to 44.0 with an average of $36.0 \pm 4.11$

There was no signs and symptoms of infection including swelling, redness, hotness, pus discharge, and pain in addition to observation for any manifestations of wound healing disturbance, as wound dehiscence.

As for the implant stability, the primary stability mean value ISQ readings for implants drilled by densah bur was $74.25 \pm$ 4.95 while that of the conventional side was $59.65 \pm 5.39$. Moreover, the secondary stability mean value for implants drilled by densah bur was $76.90 \pm 4.05$ while that of the conventional side was $68.25 \pm 5.14$. Upon further statistical analysis, there was a significant improvement in both primary and secondary stability using densah burs. (table 1, 2, and figure 4) 

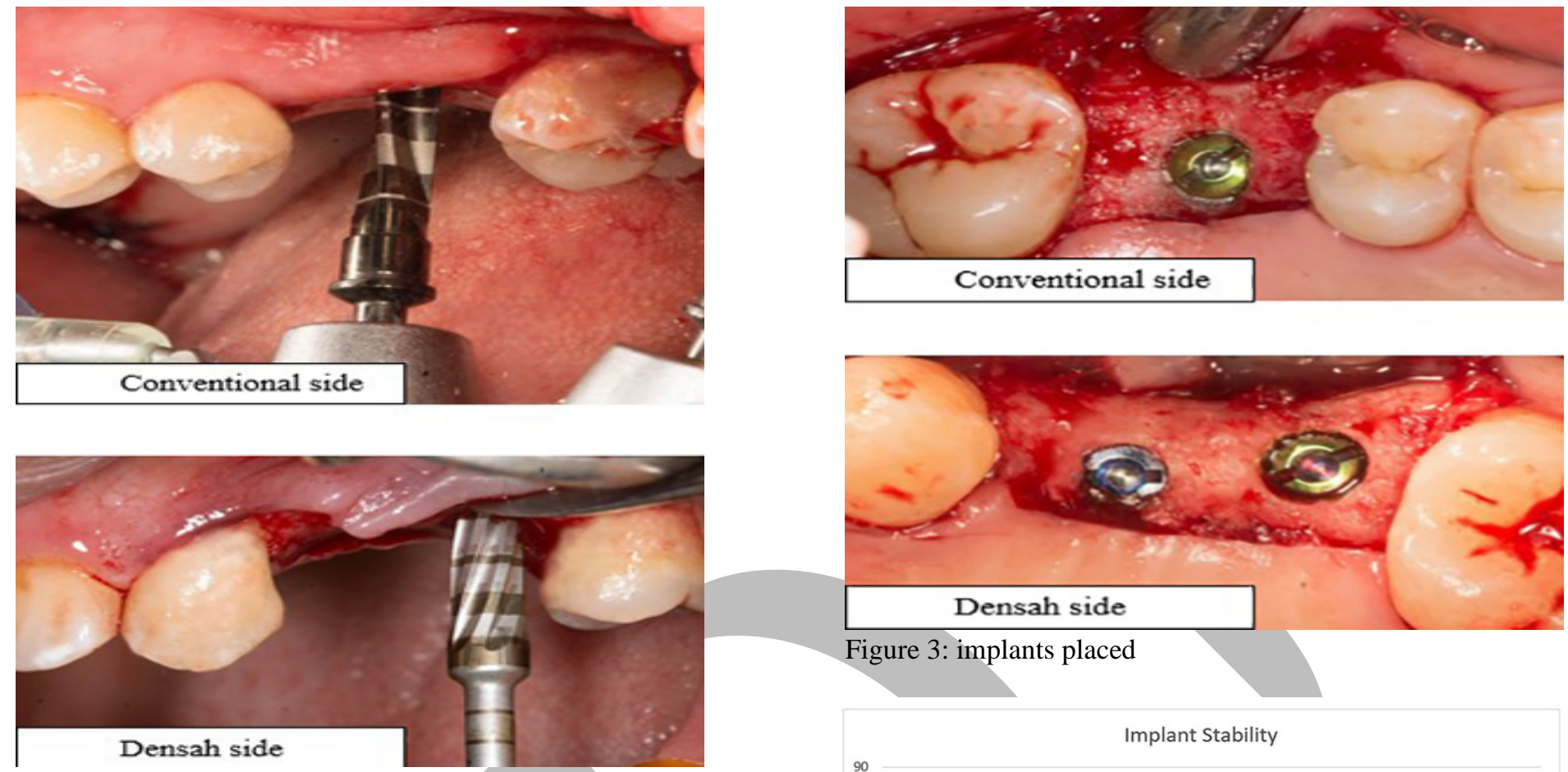

Figure 1: implant site preparation
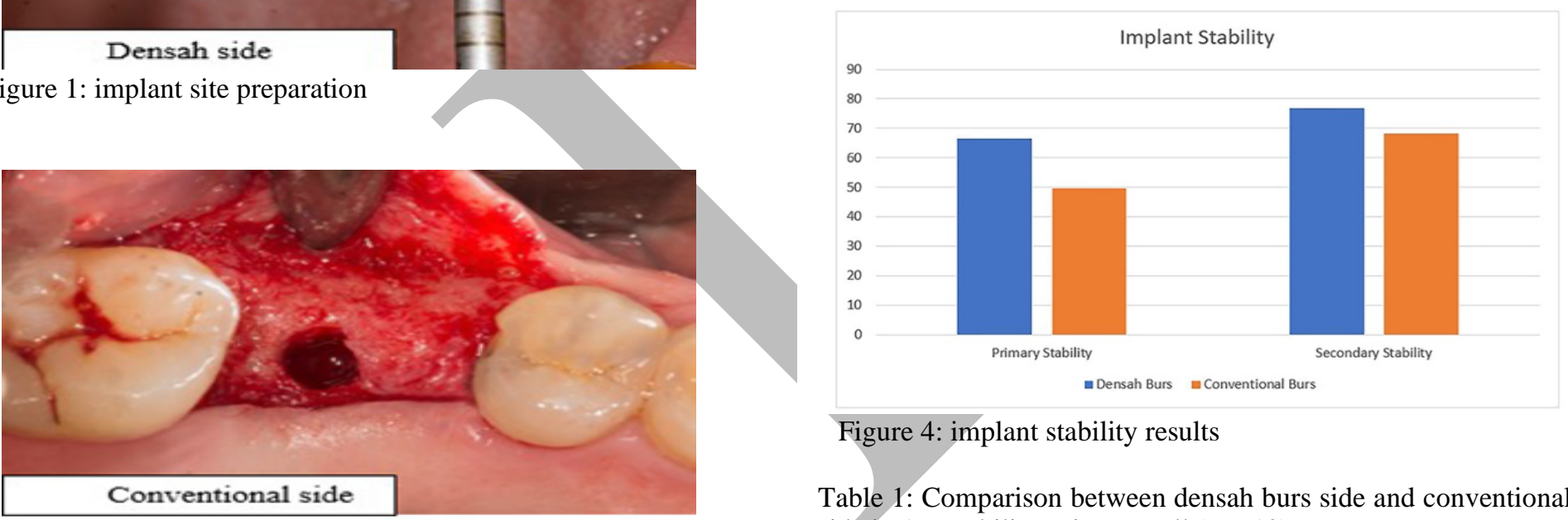

Figure 4: implant stability results

Table 1: Comparison between densah burs side and conventional side in 1ry stability using osstell $(n=10)$

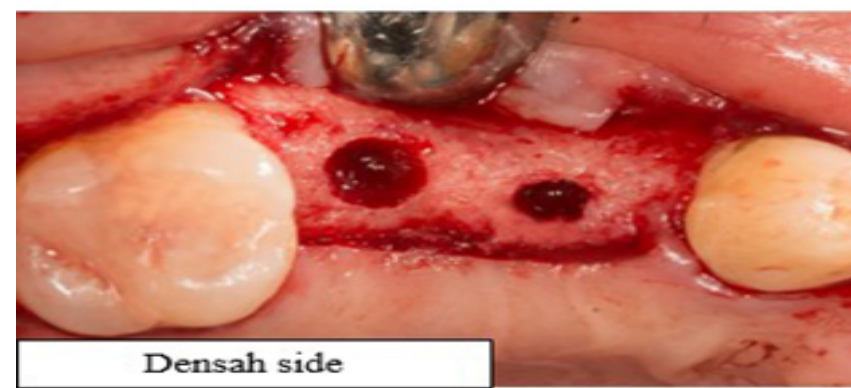

Figure 2: implant site prepared

\begin{tabular}{|c|c|c|c|c|}
\hline & $\begin{array}{c}\text { Densah burs } \\
\text { side }\end{array}$ & $\begin{array}{c}\text { Conventional } \\
\text { side }\end{array}$ & $\mathbf{t}$ & $\mathbf{p}$ \\
\hline 1ry stability & & & & \\
\hline Min. - Max. & $66.50-80.50$ & $49.50-64.50$ & & \\
Mean \pm SD. & $74.25 \pm 4.95$ & $59.65 \pm 5.39$ & $13.064^{\circ}$ & $<0.001^{\circ}$ \\
\hline Median & 75.50 & 62.0 & & \\
\hline
\end{tabular}

t: Paired t-test

p: $p$ value for comparing between the two side

*: Statistically significant at $p \leq 0.05$ 
Table 2: Comparison between densah burs side and conventional side in 2ry stability using osstell $(\mathrm{n}=10)$

\begin{tabular}{|c|c|c|c|c|}
\hline & $\begin{array}{c}\text { Densah burs } \\
\text { side }\end{array}$ & $\begin{array}{c}\text { Conventional } \\
\text { side }\end{array}$ & $\mathbf{t}$ & $\mathbf{p}$ \\
\hline 2ry stability & & & $\ldots$ & \\
\hline Min. - Max. & $71.0-83.0$ & $58.50-74.0$ & & \\
\hline Mean \pm SD. & $76.90 \pm 4.05$ & $68.25 \pm 5.14$ & $8.124^{*}$ & $<0.001^{*}$ \\
\hline Median & 77.75 & 69.25 & & \\
\hline
\end{tabular}

t: Paired t-test

p: $p$ value for comparing between the two side

*: Statistically significant at $\mathrm{p} \leq 0.05$

\section{DISCUSSION}

Ever since the commencement of dental implant therapy, the procedure employed for site preparation has been considered one of the most vital aspects affecting osseointegration. The preservation of the bone volume and histologic structure has been considered dependent on the procedures during the preparation of the implant site. Consequently, instruments for implant site preparation with the capability of enhancing osseointegration are important. $(19,21)$

This present study was conducted on ten patients in need of at least two dental implants on each side in the posterior maxillary region (premolars and molars), they were selected from the Outpatient Clinic of the Oral and Maxillofacial Surgery Department, Faculty of Dentistry, Alexandria University.

The study was conducted to compare the OD technique using the newly designed Densah burs with the conventional drills used to prepare the osteotomy site for the implant. Split-mouth design was used for this study where illustrated that the main purpose of the split-mouth design is to remove all components related to differences between subjects from the treatment comparisons, by making within-patient Comparisons, rather than between-patient comparisons. (22)

. In this study all patients in the current study were non-smokers. A study by Clementini et al concluded that exposure to smoking has a harmful effect on the peri-implant bone loss that eventually leads to implant failure. (23)

All cases in the present study were selected free from parafunctional habits such as bruxism and clenching, which increase the magnitude of the forces. In such patients, the duration of the forces are extensive and their direction is more horizontal than axial to the implants, which leads to mechanical complications and failure of implants according to Manfredini et al in 2014. (24)

In the current study, there no patient exhibited signs and symptoms of infection. Maintaining sterile conditions during the surgical procedure was of utmost importance. Saliva, perioral skin, unsterile instruments, contaminated gloves, operating room air, or air expired by the patient, all interfere in the surgical procedure leading to contamination of the implant site, causing infection in the implant site.(25) This measure is advisable even though a retrospective analysis by Scharf and Tarnow comparing 273 implants inserted under "sterile" conditions and 113 implants placed under "clean" conditions showed no statistically significant differences in the success rates of the two groups.(26) Various imaging options are available for the evaluation of the recipient site. In the present study, panoramic radiographs and CBCT were taken for each patient. CBCT was taken immediately post-operative and on 6th months follow-up period to measure the amount of ridge expansion, marginal bone level and to detect the changes in bone density surrounding dental implants.

In our study, CBCT was performed for all patients preoperatively. Corresponding to the studies they reported that the use of CBCT in implant dentistry vary from preoperative analysis regarding specific anatomic considerations, site development using grafts and treatment planning to postoperative evaluation. $(27,28)$, Along with, lower radiation dose, reduced costs and the relative grey density values of CBCT images making it a useful substitute for computerized tomography (CT). (29, 30)

As regards the surgical procedures, In this study it was conducted under local anesthesia all patients had a horizontal mid-crestal incision through the attached gingival for implant insertion. (31)

In-vivo study in rabbits by Kim JR et al have also demonstrated, that a crestal incision, mucoperiosteal flap elevated result in better bone implant contact (BIC) that will speed up bone remodeling and, in turn, result in early secondary implant stability and early loading. (32) However, another study by Fickl et al had demonstrated in dogs, that flap elevation results in a more pronounced loss of ridge dimension than the non-elevation of a flap. (31) This resorption and loss of alveolar bone height supposedly occur due to the rupture of the periosteum and its connective tissue insertion into the bone surface. (33)

Socket closure was performed very carefully using 3/0 silk suture material in order to prevent postoperative infection and inflammation, epithelial down growth and bone loss of alveolar crest during the healing period. (34)

In the present study, the implant stability was measured using the Resonance Frequency Analysis (RFA) via the Osstell ISQ system. RFA was chosen as a non-invasive and reliable method to assess variation in implant stability over time. RFA registrations are directly related to the stiffness of the implant in the surrounding bone: during healing an increase in implant stability quotient (ISQ)

Meredith el al were supporting this review, when stated that RFA could serve as a useful research technique and may prove to be valuable in studying the behavior of implants in surrounding tissue. In this study, a non-contacting method was used allowing the testing of the implant stability from any surface in $360^{\circ}$ around the implant fixture. $(35,36)$

In this study RFA was checked twice for both sides, immediately postoperative to measure the primary stability and 4-months post-implant placement to measure the secondary stability. The present study showed a significant increase in primary stability of $\mathrm{OD}$ technique in comparison to the conventional technique 
where the mean value of RFA was $(74.25 \pm 4.95)$ ISQ for the OD technique,compared to the mean value of the conventional drills (59.65 \pm 5.39 ) ISQ. Also the secondary stability was measured 4 month post-implant placement mean value was (76.90 \pm 4.05$)$ ISQ for the OD group and mean value was (68.25 \pm 5.14) ISQ for the conventional group which also had significant improvement for the side prepared with the OD group and as regarding bone density, there was a significant improvement for the OD over the conventional side. The same results was conducted in a study by Lehens et al tested the OD technique in vivo and reported its significant success over conventional drilling mechanically using the pull-out testing and microscopically using the histomorphometry. (12)

In other study, published that implants placed after OD method displayed a lesser "value of actual micromotion" (VAM) in comparison to implants placed following typical drilling. which also support our results. (37)

However, in this study, implant stability was measured two months after the implant placement not immediately, which is an unsuitable time for calculating stability. It should also be noted that implants used in the control group had a narrower diameter than those used in the OD group (3.8 mm vs. $5.0 \mathrm{~mm}$ ). Although the researchers claim that variable implant surfaces areas should not have extreme effect on VAM, they ignored to compute how this difference in diameter affects VAM or lateral stiffness. With the records provided, a 58\% difference in lateral stiffness was calculated as a result of diameter difference alone, with the narrower diameter implant having lesser lateral stability. Consequently, thr assumption that "osseodensification” rises an implant's primary stability is questionable.

\section{CONCLUSIONS}

Within the limitation of this study, the following could be concluded:

- There was an improvement in the primary and secondary stability using the densah drills compared to conventional drilling technique.

\section{Conflict of interest}

The authors declare that they have no conflicts of interest.

\section{REFERENCES}

1. Meredith N. Assessment of implant stability as a prognostic determinant. Int J Prosthodont. 1998;11(5).

2. Marquezan M, Osorio A, Sant'Anna E, Souza MM, Maia L. Does bone mineral density influence the primary stability of dental implants? A systematic review. Clin Oral Implants Res. 2012;23(7):767-74.

3. Hao Y, Zhao W, Wang Y, Yu J, Zou D. Assessments of jaw bone density at implant sites using 3D cone-beam computed tomography. Eur Rev Med Pharmacol. 2014:6.

4. Lee SH, Kim YS, Kim WK, Lee YK. Immediate implant placement in areas of aesthetic priority. J Korean Acad Periodontol. 2007;37(3):637-46.

5. Meredith N. Assessment of implant stability as a prognostic determinant. Int J Prosthodont. 1998;11(5):491-501.
6. Turkyilmaz I, Aksoy U, McGlumphy EA. Two alternative surgical techniques for enhancing primary implant stability in the posterior maxilla: a clinical study including bone density, insertion torque, and resonance frequency analysis data. Clin Implant Dent Relat Res. 2008;10(4):231-7.

7. Degidi M, Daprile G, Piattelli A. Influence of underpreparation on primary stability of implants inserted in poor quality bone sites: an in vitro study. J Oral Maxillofac Surg. 2015;73(6):1084-8.

8. Tabassum A, Walboomers XF, Wolke JG, Meijer GJ, Jansen JA. Bone particles and the undersized surgical technique. J Dent Res. 2010;89(6):581-6.

9. Tabassum A, Meijer GJ, Walboomers XF, Jansen JA. Evaluation of primary and secondary stability of titanium implants using different surgical techniques. Clin Oral Implants Res. 2014;25(4):487-92.

10. Kanathila H, Pangi A. An Insight into the Concept of Osseodensification-Enhancing the Implant Stability and Success. J Clin Diagn. 2018;12(7):1-3.

11. Asnani K. Deceiving the Uncertainty of Primary Stability: Densah [R] Burs. J Int Clin Dent Res Organ. 2017;9(2):52-.

12. Lahens B, Neiva R, Tovar N, Alifarag AM, Jimbo R, Bonfante EA, et al. Biomechanical and histologic basis of osseodensification drilling for endosteal implant placement in low density bone. An experimental study in sheep. J Mech Behav Biomed. 2016;63:56-65.

13. Hofbauer A, Huwais S. Osseodensification facilitates ridge expansion with enhanced implant stability in the maxilla: part II case report with 2-year follow-up. Implant Practice. 2015;8(2):14-21.

14. Porter JA, von Fraunhofer JA. Success or failure of dental implants? A literature review with treatment considerations. General dentistry. 2005;53(6):423-32.

15. Nagarajan A, Perumalsamy R, Thyagarajan R, Namasivayam A. Diagnostic imaging for dental implant therapy. J Clin Imaging Sci. 2014;4(Suppl 2):4.

16. Al-Juboori MJ, AbdulRahaman SB. The Effect of Flapless and Full-thickness Flap Techniques on Implant Stability During the Healing Period. The open dentistry journal. 2015;9:243-9.

17. Ahmad N, Saad N. Effects of antibiotics on dental implants: a review. Journal of clinical medicine research. 2012;4(1):1-6.

18. Larjava H. Oral wound healing: Cell Biology and Clinical Management: John Wiley \& Sons; 2012.

19. Huwais S, Meyer EG. A Novel Osseous Densification Approach in Implant Osteotomy Preparation to Increase Biomechanical Primary Stability, Bone Mineral Density, and Bone-to-Implant Contact. Int J Oral Maxillofac Implants. 2017;32(1):27-36.

20. Han J, Lulic M, Lang NP. Factors influencing resonance frequency analysis assessed by Osstell mentor during implant tissue integration: II. Implant surface modifications and implant diameter. Clin Oral Implants Res. 2010;21(6):60511.

21. Albrektsson T, Branemark PI, Hansson HA, Lindstrom J. Osseointegrated titanium implants. Requirements for 
ensuring a long-lasting, direct bone-to-implant anchorage in man. Acta orthopaedica Scandinavica. 1981;52(2):155-70.

22. Zanatta RF, da Silva TM, Esper MALR, Bresciani E, Caneppele TMF, de Paiva Gonçalves SE. Guidelines for conducting split-mouth clinical studies in restorative dentistry. Brazilian Dental Science. 2017;20(2):29-37.

23. Clementini M, Rossetti P, Penarrocha D, Micarelli C, Bonachela W, Canullo L. Systemic risk factors for periimplant bone loss: a systematic review and meta-analysis. Int J Oral Maxillofac Surg. 2014;43(3):323-34.

24. Manfredini D, Poggio CE, Lobbezoo F. Is bruxism a risk factor for dental implants? A systematic review of the literature. Clin Implant Dent Relat Res. 2014;16(3):460-9.

25. Kate MA, Palaskar S, Kapoor P. Implant failure: A dentist's nightmare. J Dent Implant. 2016;6(2):51.

26. Scharf DR, Tarnow DP. Success rates of osseointegration for implants placed under sterile versus clean conditions. J Periodontol. 1993;64(10):954-6.

27. Cassetta M, Sofan AA, Altieri F, Barbato E. Evaluation of alveolar cortical bone thickness and density for orthodontic mini-implant placement. J Clin Exp Dent. 2013;5(5):e245.

28. Bornstein MM, Scarfe WC, Vaughn VM, Jacobs R. Cone beam computed tomography in implant dentistry: a systematic review focusing on guidelines, indications, and radiation dose risks. Int $\mathrm{J}$ Oral Maxillofac Implants. 2014;29:55-77.

29. Pedroso LAdM, Garcia RR, Leles JLR, Leles CR, Silva MAGS. Impact of cone-beam computed tomography on implant planning and on prediction of implant size. Braz Oral Res. 2014;28(1):46-53.

30. Chasioti E, Sayed M, Drew H. Novel techniques with the aid of a staged CBCT guided surgical protocol. Case Rep Dent. $2015 ; 2015$.
31. Fickl S, Zuhr O, Wachtel H, Bolz W, Huerzeler MB. Hard tissue alterations after socket preservation: an experimental study in the beagle dog. Clin Oral Implants Res. 2008;19(11):1111-8.

32. Kim J-R, Kim S-H, Kim I-R, Park B-S, Kim Y-D. Low-level laser therapy affects osseointegration in titanium implants: resonance frequency, removal torque, and histomorphometric analysis in rabbits. J Korean Assoc Oral Maxillofac Surg. 2016;42(1):2-8.

33. Becker W, Wikesjö UM, Sennerby L, Qahash M, Hujoel P, Goldstein $M$, et al. Histologic evaluation of implants following flapless and flapped surgery: a study in canines. J Periodontol. 2006;77(10):1717-22.

34. Becker W. Guided tissue regeneration for implants placed into extraction sockets and for implant dehiscences: Surgical techniques and case reports. Int J Periodont Rest Dent. 1990;10:377-91.

35. Meredith N, Books K, Fribergs B, Jemt T, Sennerby L. Resonance frequency measurements of implant stability in viva. A cross-sectional and longitudinal study of resonance frequency measurements on implants in the edentulous and partially dentate maxilla. Clin Oral Implants Res. 1997;8(3):226-33.

36. Meredith N, Alleyne D, Cawley P. Quantitative determination of the stability of the implant-tissue interface using resonance frequency analysis. Clin Oral Implants Res. 1996;7(3):261-7.

37. Berardini M, Trisi P, Falco A. introduction of a new noninvasive parameter to measure implant secondary stability and osseointegration: Value of Actual Micromotion (vam). Clin Oral Implants Res. 2015;26:89. 